Relations industrielles

Industrial Relations

\title{
Studies in Social Power. Dorwin Cartwright, éditeur, Research Center for Group Dynamics, Institute for Social Research, The University of Michigan, Ann Arbor, 1959, pp. 225.
}

\section{Émile Gosselin}

Volume 17, numéro 3, juillet 1962

URI : https://id.erudit.org/iderudit/1021588ar

DOI : https://doi.org/10.7202/1021588ar

Aller au sommaire du numéro

Éditeur(s)

Département des relations industrielles de l’Université Laval

ISSN

0034-379X (imprimé)

1703-8138 (numérique)

Découvrir la revue

Citer ce compte rendu

Gosselin, É. (1962). Compte rendu de [Studies in Social Power. Dorwin Cartwright, éditeur, Research Center for Group Dynamics, Institute for Social Research, The University of Michigan, Ann Arbor, 1959, pp. 225.] Relations industrielles / Industrial Relations, 17(3), 355-356.

https://doi.org/10.7202/1021588ar

Tous droits réservés (C Département des relations industrielles de l’Université Laval, 1962
Ce document est protégé par la loi sur le droit d'auteur. L'utilisation des services d’Érudit (y compris la reproduction) est assujettie à sa politique d'utilisation que vous pouvez consulter en ligne.

https://apropos.erudit.org/fr/usagers/politique-dutilisation/ 
dépendra de son comportement. L'on comprend mieux la structure et la fonction d'une organisation si l'on analyse de quelle façon les décisions et le comportement de tels employés sont influencés au sein de l'organisation.

L'étude porte sur le service de protection des forêts, une division du Département de l'Agriculture des E.-U. Après avoir fait une longue analyse de l'ampleur et de la complexité des divers services forestiers, l'auteur étudie les problèmes majeurs qui se posent: problèmes de communication dus à la distance, à l'interprétation des lois et règlement., relations avec le public, sélection des officiers, déconcentration des services. Si de tels problèmes n'étaient pas surveillés de près, l'ensemble de l'organisation aurait vite tendance à se fragmenter, et elle cesserait d'agir comme un tout fonctionnel et bien intégré. Or l'auteur démontre comment les divers facteurs de désintégration sont maintenus sous contrôle et les politiques mises en oeuvre pour faire du service une organisation travaillant de façon harmonieuse et bien coordonnée. Il analyse de quelle façon les politiques sont élaborées, quelles soient d'ordre financier ou purement administratif, et comment l'on détecte et corrige toute déviation aux normes, une fois qu'elles auront été établies. Enfin, l'auteur démontre qu'il n'est pas suffisant pour les membres d'une organisation de posséder uniquement une compétence purement technique, mais ils doivent être capables de faire équipe et d'exécuter spontanément les programmes selon les politiques et les directives.

\section{EMme Gosselin}

Dictionnaire économique et social, par Thomas Suavet. Coll. «Initiation économique ». Economie et Humanisme. Les Editions ouvrières, 12, avenue Soeur-Rosalie, Paris (13), 1962, $455 \mathrm{pp}$.

Comme nous en avertit l'auteur, cet ouvrage n'est pas destiné aux spécialistes qui veulent accroître leurs connaissances dans leur domaine. Mais il leur permet d'ouvrir leurs horizons et de trouver certains éléments utiles à une meilleure compréhension de l'ensemble du champ économique et social.

L'auteur a réussi à éviter deux extrèmes: présenter un simple compendium de mots choisis dans Larousse ou Quillet, d'une part, et, d'autre part, de faire une encyclopédie. Autour d'environ trois cents mots-clés, il a synthétisé un ensemble de notions théoriques et de données factuelles dont la connaissance est indispensable à tous ceux qui travaillent dans les domaines social, économique ou politique. La plupart des articles sont suivis d'une brève bibliographie. A la fin de l'ouvrage, on trouve un répertoire alphabétique des mots analysés soit dans un article spécial ou dans un autre indiqué par un renvoi.

L'auteur s'est donné beaucoup de peine pour présenter un instrument de travail à la fois bref et abordable. Parce que cet ouvrage a été préparé pour la France, les références aux problèmes concrets sont illustrées soit par l'histoire, la législation et les institutions de ce pays. Si l'auteur avait voulu tenir compte des lecteurs en dehors de la France, il aurait été obligé d'accroître considérablement le format de son dictionnaire et de faire appel à la collaboration de plusieurs étrangers d'expression française. Même si pour cette raison, les lecteurs canadiens ne peuvent tirer autant de bénéfices que les lecteurs français, ils trouveront encore suffisamment avantage à posséder un tel ouvrage et à le consulter. Tout ce que nous pouvons souhaiter, c'est qu'un jour soit publié un supplément pour le Canada et les autres pays d'expression française.

\section{GÉrard Dion}

Studies in Social Power. Dorwin Cartwright, éditeur, Research Center for Group Dynamics, Institute for Social Research, The University of Michigan, Ann Arbor, 1959, pp. 225.

Ce volume représente le résultat de travaux et de recherches qui se sont étendus sur une période de huit années. Chaque chapitre étudie un aspect déterminé du pouvoir dans la société, soit en tant que cause ou encore en tant qu'effet. Par exemple: quels sont les sources ou les déterminants du pouvoir? Sous quelles conditions la possession du pouvoir devient-elle de fait l'exercice du pouvoir! Comment le pouvoir affecte$t$-il les communications entre personnes ? Le pouvoir constitue-t-il une menace pesant sur ceux qui l'exercent! Quels sont les effets de la possession du pouvoir sur 
les relations entre le détenteur du pouvoir et les autres?

Cet ouvrage, fruit du travail de onze chercheurs différents n'entend pas constitué un tout théorique intégré. A cause de la variété des approches employées par les différents auteurs, l'on trouvera difficilement un cadre conceptuel qui serait le même dans tous ces travaux. Par exemple, chaque auteur procède à partir d'une définition du pouvoir qui lui est propre. Mais, dans l'ensemble, ce qui unifie les travaux, c'est une préoccupation de pénétrer plus à fond un ensemble de problèmes connexes: Comment une personne ou un groupe peuvent-ils influencer les attitudes et les comportements des autres?

Après avoir souligné, dans un premier chapitre, que les psychologues sociaux, praticiens, théoriciens et chercheurs, ont trop négligé le problème du pouvoir en société, un chercheur conclut que les problèmes fondamentaux en psychologie sociale ne sauraient se résoudre sans une attention plus considérable portée aux divers aspects du pouvoir. Les trois chapitres subséquents traitent des con- séquences de l'exercice du pouvoir pour ceux qui en sont l'objet. Les quatre chapitres suivants traitent des circonstances entourant l'établissement de relations de pouvoir au sein d'un groupe contrôlé en laboratoire et au sein de groupes naturels. Enfin, les trois derniers chapitres traitent de la théorie des relations de pouvoir. Quels sont les types et les sources de pouvoir? Qu'estce qui influence les opinions et les attitudes au sein d'un groupe? Comment situer une théorie du pouvoir dans le contexte d'une théorie globale du comportement humain?

Le travail s'adresse plus particulièrement aux spécialistes de la psychologie sociale. Il est l'oeuvre de professeurs et chercheurs rattachés au « Research Center for Group Dynamics » de l'Université de Michigan. Le travail est très bien présenté et s'accompagne d'une bibliographie fort riche, ce qui, à lui seul, devrait inciter ceux qui s'intéressent aux «relations humaines » ou à la psychologie sociale à recourir fréquemment à cet ouvrage.

Emile Gosselin

\section{PUBLICATIONS RÉCENTES RECENT PUBLICATIONS}

\section{Généralités}

«Organos centrales de planification 》, E.C.A., Ano IV, No. 10, Marzo 1962, pp. 7-22.

Revue de l'Action Populaire, no 160, juillet-août 1962 .

- Les démocraties qui fonctionnent 》, par G. Lavau, pp. 785-794.

- Pouvoir et liberté politiques», par A. Jeannière, pp. 795-806.

—Qu'est-ce que la polyarchie?», par Fr. Bourricaud, pp. 807-821.

«Thomas J. Hagerty, the Church and Socialism », by Robert E. Doherty, Labor History, Vol. 3, No. 1, Winter 1962, pp. 39-56.
«Work Injuries and Recovery 》, by Earl F. Cheit, Monthly Labor Review, October and November 1961, pp. 1059-120.5.

«Medical Expenses and Choice of Plans: A Case Study », by Burton Wolfman, Monthly Labor Review, October and November 1961, pp. 1186-1190.

* Cima Stresses Management Education », Plant Administration and Engineering, Vol. 22, No. 7, July 1962, p. 44.

\&Le Concile et les chefs d'entreprise chrétiens », par Mgr Garrone, Documents et Commentaires, 68e année, no 12, mai-juin 1962, pp. 7-8.

«L'entreprise, étape d'un progrès doctrinal », par Pierre Bigo, Revue de l'Action Populaire, no 159, juin 1962, pp. 644-656. 